\section{Y-12}

NATIONAL SECURITY COMPLEX

MANAGED BY BWXT Y-12, L.L.C. FOR THE UNITED STATES DEPARTMENT OF ENERGY UCN-13672 (10-00)

\section{Monte Carlo Modeling of High-Energy Film Radiography}

\author{
A.C. Miller, Jr. \\ J. L. Cochran \\ V.E. Lamberti \\ Technology Development
}

Presented at the:

Nuclear Mathematical and Computational Sciences

Gatlinburg, Tennessee, April 6-11, 2003

Date of issue: March 28, 2003

Prepared by the Y-12 National Security Complex

P. O. Box 2009

Oak Ridge, Tennessee 37831-8169

managed by

BWXT Y-12 L.L.C.

for the

U.S. DEPARTMENT OF ENERGY Under contract DE-AC05-00OR-22800 


\section{DISCLAIMER}

This report was prepared as an account of work sponsored by an agency of the United States Government. Neither the United States Government nor any agency thereof, nor any of their employees, makes any warranty, express or implied, or assumes any legal liability or responsibility for the accuracy, completeness, or usefulness of any information, apparatus, product, or process disclosed, or represents that its use would not infringe privately owned rights. Reference herein to any specific commercial product, process, or service by trade name, trademark, manufacturer, or otherwise, does not necessarily constitute or imply its endorsement, recommendation, or favoring by the United States Government or any agency thereof. The views and opinions of authors expressed herein do not necessarily state or reflect those of the United States Government or any agency thereof.

\section{COPYRIGHT NOTICE}

"The submitted manuscript has been authored by a contractor of the U.S. Government under contract DE-AC05-00OR22800.

Accordingly, the U.S. Government retains a paid-up, nonexclusive, irrevocable, worldwide license to publish or reproduce the published form of this contribution, prepare derivative works, distribute copies to the public, and perform publicly and display publicly, or allow others to do so, for U.S. Government purposes." 


\title{
MONTE CARLO MODELING OF HIGH-ENERGY FILM RADIOGRAPHY
}

\author{
Arthur C. Miller, Jr., Joseph L. Cochran, and Vincent E. Lamberti \\ BWXT Y-12, L.L.C. \\ PO Box 2009 , Oak Ridge, Tenn 37831 \\ milleracjr@y12.doe.gov; cochranj1@y12.doe.gov, lambertive@y12.doe.gov
}

\begin{abstract}
High-energy film radiography methods, adapted in the past to performing specific tasks, must now meet increasing demands to identify defects and perform critical measurements in a wide variety of manufacturing processes. Although film provides unequaled resolution for most components and assemblies, image quality must be enhanced with much more detailed information to identify problems and qualify features of interest inside manufactured items. The work described is concerned with improving current $9 \mathrm{MeV}$ nondestructive practice by optimizing the important parameters involved in film radiography using computational methods. In order to follow important scattering effects produced by electrons, the Monte Carlo N-Particle (MCNP) transport code was used with advanced, highly parallel computer systems. The work has provided a more detailed understanding of latent image formation at high X-ray energies, and suggests that improvements can be made in our ability to identify defects and to obtain much more detail in images of fine features.
\end{abstract}

Key Words: MCNP, X-ray,modeling,radiography, high-energy

\section{INTRODUCTION}

Film radiography methods have been optimized for specific imaging tasks through considerable trial-and-error efforts based on incomplete scientific guidance. These methods are now expected to meet increasing demands in support of manufacturing processes. At Y-12, a retiring workforce, downsizing pressures, and new regulatory requirements indicate that supplemental technologies must be implemented to cost-effectively meet new requirements. Tomographic inspection methods are being used more widely in medical imaging applications and are generally much easier to interpret than conventional radiographs. In spite of its ease of interpretation, tomography cannot be readily applied to high-energy radiography needs, particularly those involving objects containing a wide range in density.

Computer modeling and simulation is being applied to parametrically optimize key elements in the radiograph process. The overall goal is to achieve sharper images with higher contrast in dim areas through adjustments of several important variables. Initial studies in MCNP modeling of the high-energy X-ray film process have been previously reported [1]. 


\section{COMPUTER MODELING AND SIMULATION}

Although the MCNP (Monte Carlo N-Particle) code is a powerful scientific tool, its computational requirements can easily become staggering when applied to realistic problems. Following billions of particles to achieve adequate statistics is particularly challenging when only a relatively small number survive. Various methods are available within MCNP to improve the statistical confidence in the results, but each technique must be carefully evaluated and applied with care. In general, the approach taken here was to use large numbers of incident photons to achieve statistically significant numbers of particles that could interact with the film and employ parallel computing machines to reduce long run times.

Version MCNP4c was used in the simulations. It handles several scatter mechanisms and is able to separate electron and photon effects. Similar codes have found use in medical research and have been characterized and compared with MCNP [2,3]. A simplified radiographic process is described by Figure 1 .

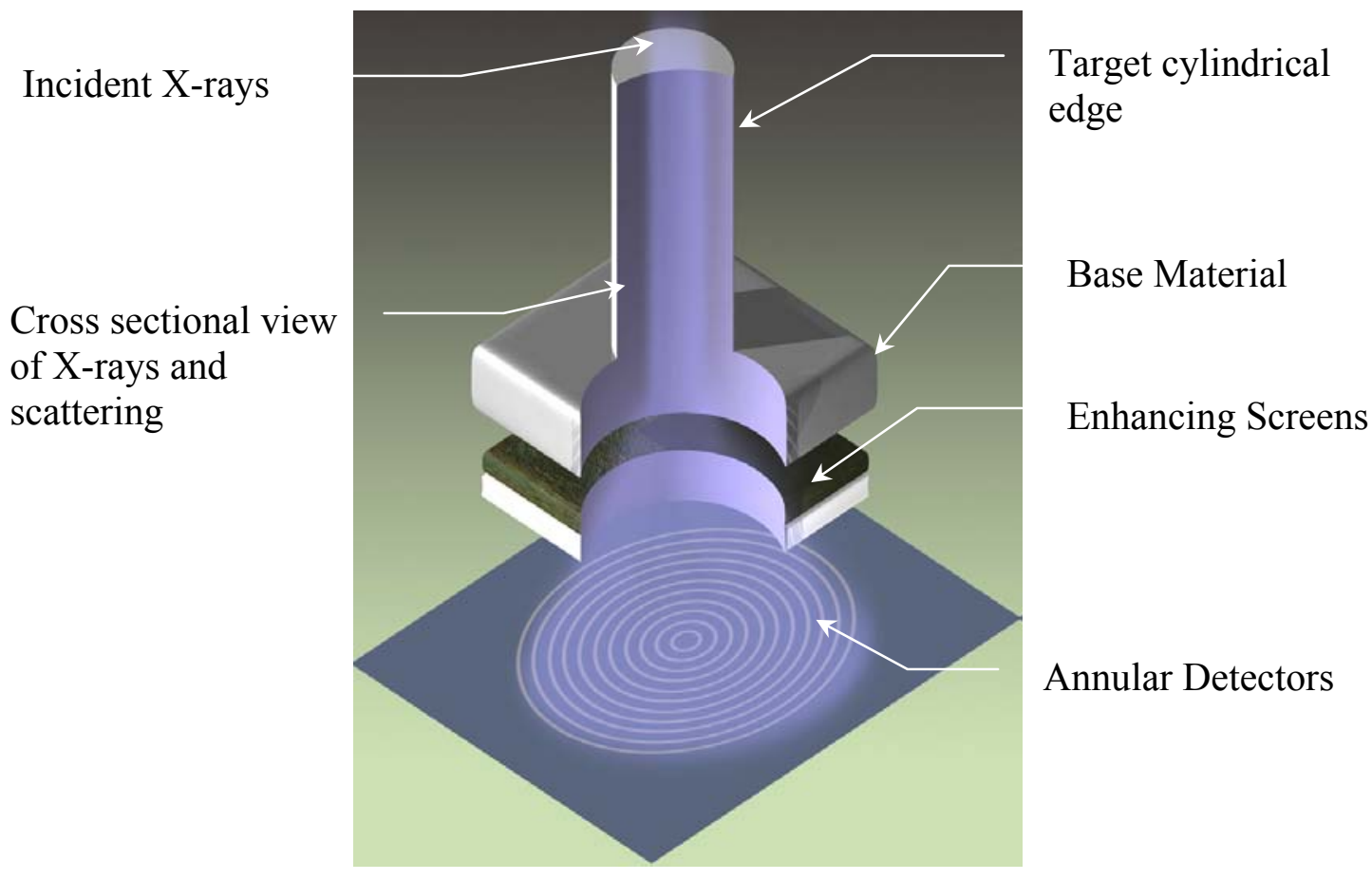

Figure 1. Model setup for edge response function

Photons from a $9 \mathrm{MeV}$ Bremsstrahlung spectrum, idealized collimated source were modeled as passing through a dense object simulating an edge and then interacting with a screen/detector system. The object, modeled as uranium, introduces the proper beam hardening and particle scattering effects. Photoelectric, Compton, and pair production effects were tracked in simulations of high density objects like tungsten and uranium. The spread of the extended beam by scattering as it falls across the step target generates the edge or line spread function as shown 
in Figure 2. Lead enhancing screens were used in the modeling, but earlier simulations showed that multilayer screens could be made using gold foil in conjunction with a thick, low atomic number attenuating layer to enhance images. Enhancing screens perform two important functions in the radiographic process: First, interactions in the screen release lower energy electrons that efficiently expose the film, and second, screens attenuate low energy scattered radiation that efficiently exposes, but merely contributes "fog", not additional information to the film. Concentric, annular detector areas in the film plane are normalized to reflect equal areas and are analyzed for the total energy deposited. The geometry is circularly symmetric to improve counting statistics.

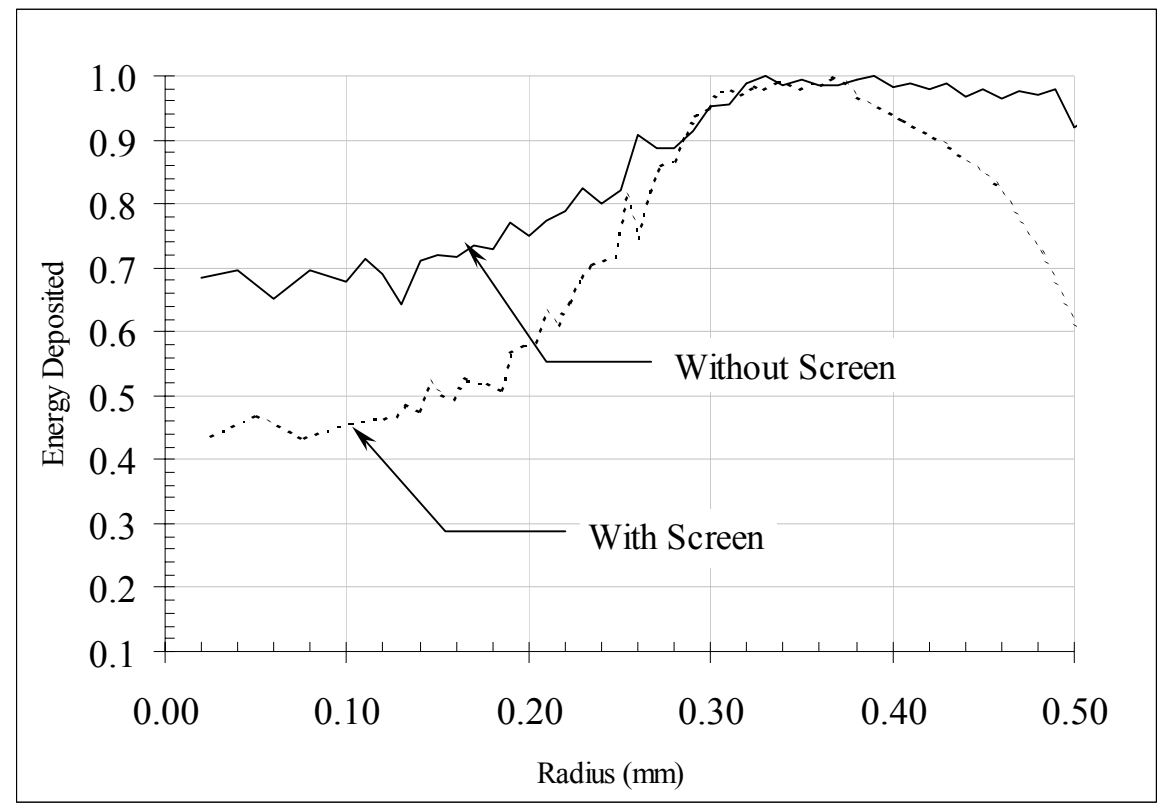

Figure 2. MCNP Edge response function

An MCNP "F8" tally was used to record the deposited energy absorbed by the film detectors. In spite of long run times (70 hours with a single processor workstation) and large numbers of incident photons, the data show considerable variability in the response due to statistical fluctuations. Long run times are necessary since the energy deposited in the film from the surviving electrons dominates the line spread.

Analysis of the edge response can be easily related to the resolving capabilities of a system. Earlier studies have discussed the results of Modulation Transfer Function analysis of resolving capability [1]. Effects of the enhancement screen commonly used in radiography are evident in the slope and level differences of the model with and without the screen. The drop-off in the "with screen" condition was caused by failing to extend the source sufficiently past the edge of the high density region in the target of Figure 1. Response with the enhancement screen shows a somewhat sharper edge implying better image definition and a larger data range which indicates superior contrast. 
Several additional line spread tests were conducted to investigate contrast as a function of the edge width and depth. The derivative of the line spread function was calculated and a "visibility" factor was defined to be the ratio of the peak height to the full width at half-maximum. Therefore higher visibility results required sharper slope as well as higher amplitude. Results of the analysis are seen in Figure 3.

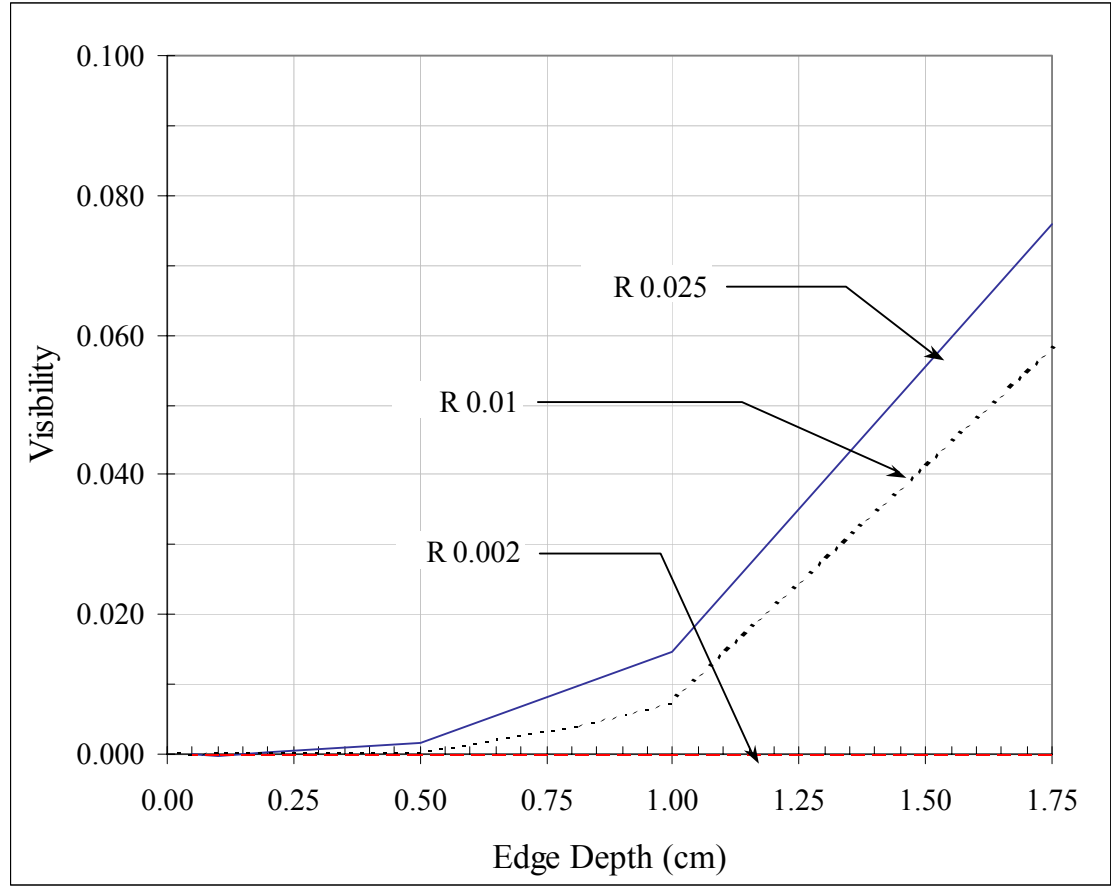

Figure 3. Visibility of various radius edges

Three different radii of the cylinder were used in the simulations at a series of cylinder heights. With a radius of $0.025 \mathrm{~cm}(0.010$ inch), visibility disappears at a 20 to 1 ratio between the edge radius and edge depth. Visibility also quickly deteriorates for $0.01 \mathrm{~cm}(0.005$ inch $)$ edges. 0.002 $\mathrm{cm}$ or 0.001 inch edges were not visible even for very thick transitions. These results tend to confirm the difficulty in observing fine features using high-energy radiography and provide a framework for system resolution optimization.

A more involved model, shown in Figure 4, was developed to study the process parameters involved in imaging a small gap in an iron artifact. The simulation was designed to test the correlation between the computational model and an actual digitized film radiograph of the object under the same conditions. The cut-away view in Figure 4 shows the incident X-ray radiation scattering throughout the base material and interaction with the enhancing screens. Uranium was again used as the base material with the same lead enhancing screen as in Figure 1. In the simulation, the gap was $0.005 \mathrm{~cm}(0.002 \mathrm{in})$ wide and produced a large amount of scattering around the gap area. The film was modeled as a series of strips running parallel to the gap direction to improve counting statistics for this geometry. 


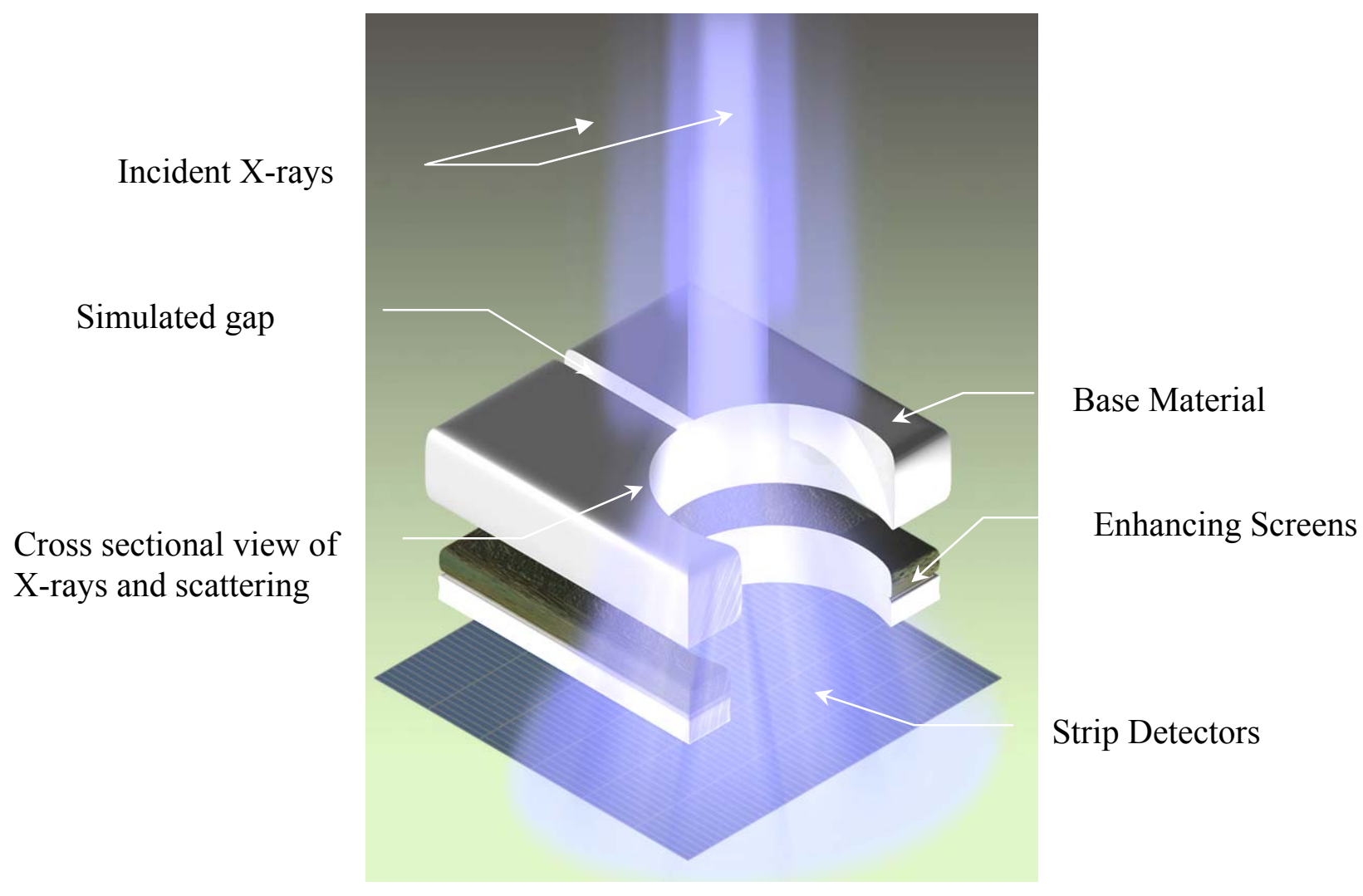

Figure 4. MCMP model setup for gap analysis

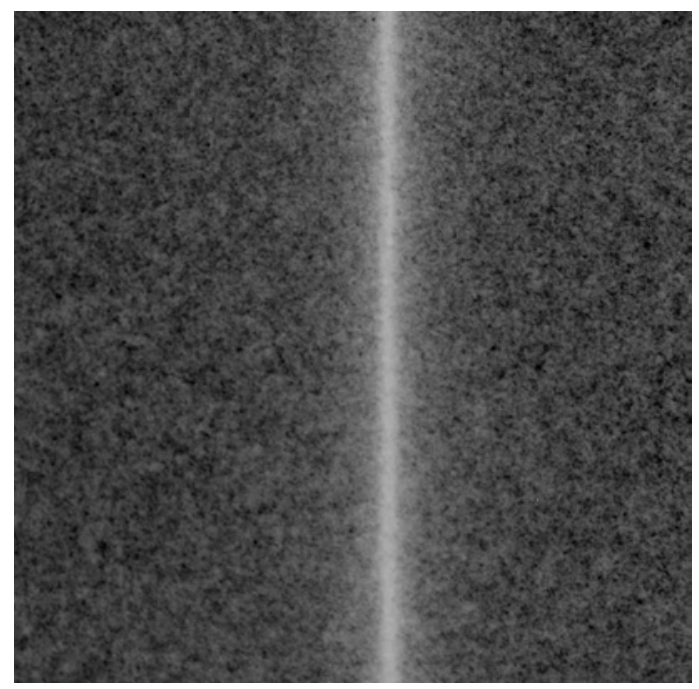

Figure 5. Actual radiograph image of an iron gap 
The actual digitized radiograph, shown in Figure 5, was taken under the exact conditions of the model and shows considerable scatter along the gap edges. The radiograph was digitized and scaled to fit the output of the MCNP simulation.

Figure 6 indicates that even though agreement with the simulation is quite good, the theoretical data predicts less broadening of the gap image than appears in the actual radiograph. Other small effects such as screen and film scatter and X-ray vault scatter may need to be incorporated for the model to more closely match experimental results.

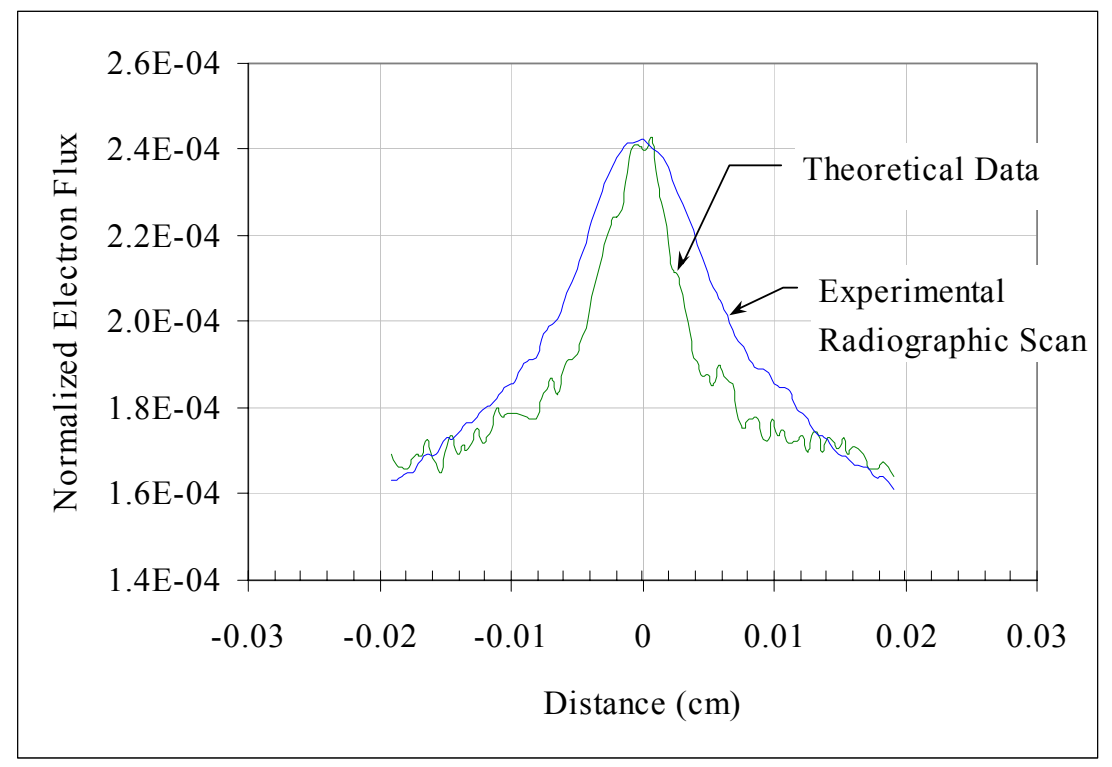

Figure 6. Comparison between MCNP and radiograph scan

A subsequent application of the gap model was used to evaluate the image quality of doublesided film. MCNP has the ability to separate the images on front and back emulsions, thereby allowing a relative comparison of the benefit of using the extra emulsion layer. As can be seen in Figure 7, the back image adds about as much scatter as additional information and brightness to the composite radiograph and is of limited use. The two emulsion layers are separated by as much as $0.018 \mathrm{~cm}(0.007 \mathrm{in})$ by a plastic substrate material that produces considerable scattering and attenuation of important imaging electrons.

Based on the modeling results, the substrate of the film also absorbs many of the electrons produced in the enhancement screen. Those surviving have scattered from their original trajectories to produce a blurred image much similar to the situation when close contact is not maintained between the enhancement screen and the film. 


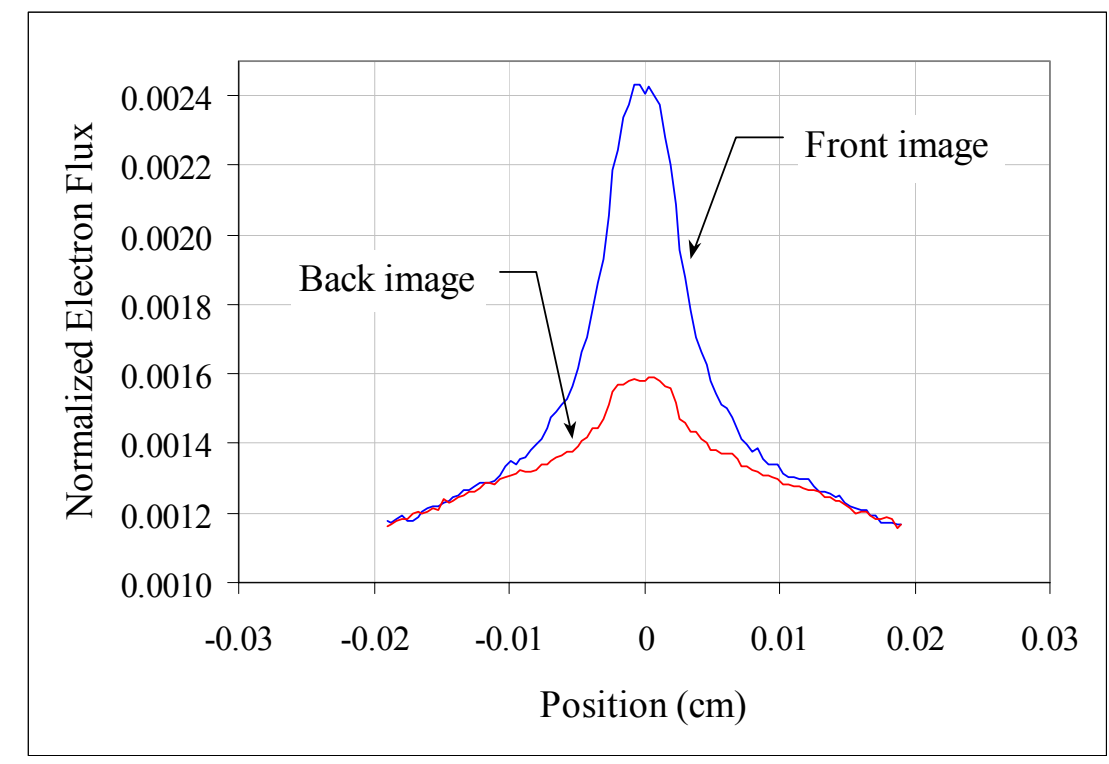

Figure 7. Double sided film images of gap

\section{MASSIVELY PARALLEL COMPUTER SIMULATIONS}

MCNP simulations were initially performed upon ASCI Manhattan, a 50 Gflop SGI Origin 2800 system located at the Oak Ridge, Tennessee Y-12 campus. Using 88 otherwise unburdened processors, simulations tracking $2 \times 10^{9}$ particles typically required about 250 hours, limiting the frequency of experiments to $2-3$ per month. In an effort to make our work more efficient, we have begun moving the calculations to the more powerful ASCI machines maintained at the national laboratories. Here, we describe some preliminary, unoptimized experiments using the 6,144-processor ASCI Blue Mountain computer at Los Alamos National Laboratory (LANL).

Mostly delivered to LANL in the second half of 1998, Blue Mountain has the symmetric multiprocessor cluster architecture commonly found on very large parallel systems [4]. The symmetric multiprocessor cluster architecture is a hybrid of two basic multiprocessor organizations: the shared memory or symmetric multiprocessor (SMP) architecture, in which each processor has access to all locations in a global shared memory using standard load operations over a single system bus, and the distributed memory architecture, in which a smaller local memory is attached to each processor and communication between processor-memory units is performed over an interconnection network.

An SMP cluster is essentially a distributed memory machine in which each processor-memory unit has been replaced by a symmetric multiprocessor. This design exploits both the high parallel efficiency achievable within an SMP cluster, a consequence of the local rather than networkbased sharing of data, and the enormous scalability of a distributed memory machine. (Contention among the processors for control of the bus usually imposes a practical limit of 128 processors for an SMP). ASCI Blue Mountain consists of 48 SGI Origin 2800 SMPs, each containing 128 250-Mhz processors; communication among the SMPs is accomplished through a 
message-passing mechanism. Jointly, this architecture represents a theoretical peak performance of 3.072 Tflop; the maximum observed LINPACK performance is 1.698 Tflop [5].

Figure 8 displays the execution time on Blue Mountain of an MCNP test program tracking $50 \times$ $10^{6}$ particles as a function of the number of processors. The test program considered a single ray of particles interacting with a dense object and a subsequent lead screen before depositing energy into annular detectors and, again, took into account photoelectric, Compton, and pair production effects.

The figure demonstrates that the advantages of parallel execution begin to decline sharply above about 128 processors. Imperfect scalabilities of this type are very frequently observed and, in general, have three principal origins: $(i)$ The application may have a large fraction of code which must execute sequentially; (ii) the application may require a great deal of communication or coordination; and (iii) the loading of the processors may be very poorly balanced [6 ].

Generally, fixed source problems as reported here do not exhibit significant sequential execution and scale very well with the number of processors. However, these runs were not optimized, and it is important to control communication between processors within MCNP in the form of dumps and rendezvous. Also, the number of particles traced per processor should be optimized with respect to the total number of processors.

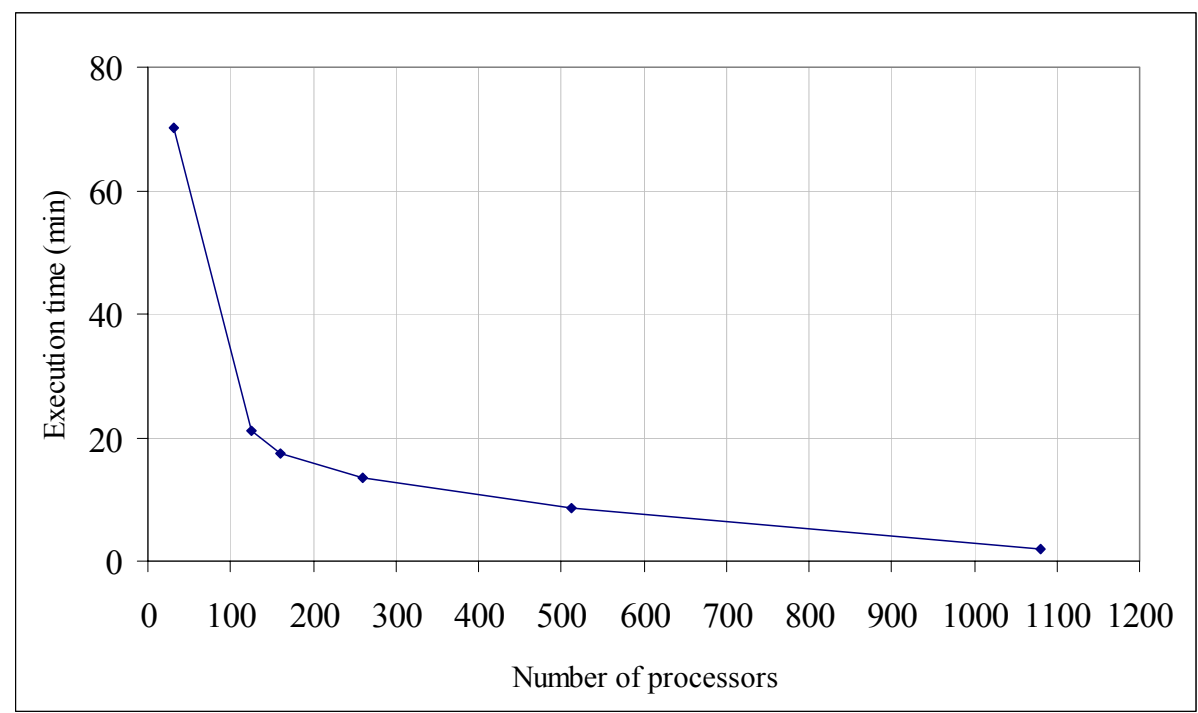

Figure 8. Execution Time vs. Processors on Blue Mountain

\section{CONCLUSIONS}

A variety of scientific models have been developed to study image formation by high-energy Xrays in radiographic film. This effort provides much needed insight into a largely skill-based 
technology by examining complex scattering mechanisms and their effect on latent image formation and ultimate quality.

The models were used to show the theoretical limits of feature visibility for a given radiographic setup and part composition. In general, these computational models allowed careful study of electron interaction with film under ideal conditions. Room scatter of low energy X-rays, film absorption, and collimation effects can all be easily eliminated in digital models, leaving a bestcase scenario. The studies were useful in optimizing complex radiographic setups since, in many cases, high-energy images may be fundamentally limited in resolution and contrast.

Testing showed that the Monte Carlo method has significant limitations as a useful tool for studying computer aided design representations of actual parts in high-energy X-ray setups. Indeed, long run times are experienced even for much simplified models such as edges and gaps. In high-energy radiography simulations, the problems typically involve deep penetration of dense materials or very thick sections of metals like aluminum and steel. Variance reduction methods are available within MCNP that can greatly improve the simulation run times, but we have found that these methods must be used carefully. Considerable understanding of the MCNP code and how variance reduction methods are applied is necessary, and results must be retested when the problem changes.

Initial testing with advanced, massively parallel computer systems showed that a major gain in execution speed occurs with relatively few processors. Computing power is steadily increasing, and small clusters can be readily configured for MCNP studies. Therefore, radiography problems that today take many hours of expensive resources can be moved to smaller clusters and allow study of accurate representations of parts and much more complex setups.

\section{ACKNOWLEDGMENTS}

The authors thank Forrest B. Brown at Los Alamos National Laboratory for his assistance and suggestion in performing MCNP testing on the Blue Mountain machine. This work was performed for the U.S. Department of Energy under Contract No. DE-AC05-00OR22800.

\section{REFERENCES}

1. Miller, A.C., Jr. and Cochran, J.L., "Optimization of Process Parameters in High-Energy Film Radiography," Review of Progress in Quantitative Nondesturctive Evaluation, Ames, Iowa, 16-20 July, 2000, Vol 20A, pp.499-506 (2000).

2. Boone, J.M., Buonocore, M.H., Cooper, V.N., III, " Monte Carlo validation in diagnostic radiological imaging," Medical Physics, Vol.27, no.6, pp.1294-304 (2000).

3. Siebers, J.V., Keall, P.J., Libvby, B., and Mohan, R., "Comparison of EGS4 and MCNP4B Monte Carlo codes for generation of photon phase space distribution for a Varian 2100C," Phys. Med. Biol. (UK), Vol.44, no.12, pp. 3009-26 (1999).

4. J. Dongarra, I. Foster, G. Fox, W. Gropp, K. Kennedy, L. Torczon, and A. White, ed. Sourcebook of Parallel Computing, Morgan Kaufmann, New York, USA (2003), pp. 45-49.

5. "Top500 Supercomputer Sites," http://www.top500.org (2002).

6. J. Dongarra, I. Foster, G. Fox, W. Gropp, K. Kennedy, L. Torczon, and A. White, ed. Sourcebook of Parallel Computing, Morgan Kaufmann, New York, USA (2003), pp. 57-59. 